\title{
VAN DE HULST ON ROBUST STATISTICS: A HISTORICAL NOTE
}

\author{
by W.R. van Zwet
}

\begin{abstract}
This paper provides a discussion of an unpublished set of notes written in 1942 by the Dutch astronomer H.C. VAN DE HULST. In these notes VAN DE HULST derives the asymptotic variances of $M$ estimators as well as trimmed means and concludes that the asymptotic variance of what is now called HUBER's estimator is the same as that of a trimmed mean. This conclusion is usually ascribed to BICKEL (1965). A letter written by D. VAN DANTzIG in 1943 providing a critical evaluation of. VAN DE HULST's results, adds interest to this suprisingly early contribution to the theory of robust statistics.
\end{abstract}

Key words: Robust estimation, estimation of location, trimmed mean, M-estimator, history of statistics.

\section{Introduction}

It is generally agreed that the history of modern mathematical statistics in the Netherlands begins with the work of VAN DANTZIG. Originally a pure mathematician, VAN DANTZIG turned to statistics and probability during the second world war. After the war, he did outstanding work in these areas and almost singlehandedly educated an entire generation of mathematical statisticians and probabilists. He was a tireless promoter of applied mathematics and one of the founders of the Mathematisch Centrum at Amsterdam. Those who didn't know this rather formidable man, should read HeMELRIJK's (1959) obituary as well as some of VAN DANTZIG's papers listed there.

Though mathematical statistics was more or less unknown territory for Dutch mathematicians at the time VAN DANTZIG entered the field, this was certainly not the case for Dutch physicists and astronomers. It is the purpose of this historical note to show that, in fact, they knew quite a bit about the subject at a very early date. In particular, I shall discuss an unpublished set of notes on what is now called robust statistics, written in 1942 by VAN DE HULST, then an astronomy student at Utrecht and presently professor emeritus of theoretical astronomy at Leiden. Almost as fascinating as the notes themselves is the correspondence about the results between VAN DE HULST and VAN DANTZIG, and the remarks that VAN DANTZIG pencilled in the margins of the notebook. Asked for his opinion, VAN DANTZIG complained at great length about the lack of mathematical rigor, but finally relented somewhat and tried to encourage VAN DE HULST to continue working on statistical problems. But things turned out differently. In 1944 VAN DE HULST predicted the $21 \mathrm{~cm}$ radio spectral line of hydrogen, which eventually led to the birth of radio astronomy. From there, he went on to a brilliant career in theoretical astronomy and never bothered to publish his investigation of robust statistics. I'm endebted to professor VAN DE HULST for mentioning his work to me and for making the notebook and the ensuing correspondence available. Thanks 
also go to Professor S.M. Stigler for drawing my attention to the work of DANIELl (1920).

\section{A problem of Hertzsprung}

In the issue of May 20, 1942, of the Bulletin of the Astronomical Institutes of the Netherlands, E. HERTZSPRUNG, director of the Observatory at Leiden, describes a sampling experiment to determine the variance of the trimmed mean. In connection with the determination of relative proper motions of stars in the Pleiades, HERTZSPRUNG discusses how one should assign weights to the observed values to account for differences in quality of the observations. He writes:

"The simplest way to deal with exorbitant observations is to reject them. In order to avoid special rules for onesided rejection the easy way of symmetrical rejection of the largest deviations to each side may be considered. The first question is then: How much is, in the case of Gaussian distribution of errors, the weight of the result diminished by a priori symmetrical rejection of outstanding observations? As the mathematical treatment of this question appears to be laborious beyond the needs mentioned above I gave preference to an empirical answer. On each of 12534 slips of paper was written with two decimals a deviation from zero in units of the mean error, in such a way that these deviations showed a Gaussian distribution. Thus 50 slips were marked with $.00,50$ with $+.01,50$ with -.01 etc.. Of these slips somewhat more than 1000 times 24 were picked out arbitrarily. Such 24 slips were in each case arranged according to the size of the deviation and mean squares of the sums of 24-x deviations calculated after symmetrical rejection of $x=0,2,4, \ldots, 22$ extreme values."

This paragraph should warm a statistician's heart, except that he may feel slightly uneasy about "somewhat more than 1000 " replications. And he has reason to feel uneasy: "Of all these samples of 24 exactly 1000 were picked out in such a way that the sum of all 24 deviations $(x=0)$ fairly well showed a Gaussian distribution with a mean square of $24 . "$

From a theoretical point of view, this ruins a perfectly good sampling experiment, as VAN DANTZIG was quick to point out, especially since no further information is supplied. There is no way of assessing the accuracy of the estimated variances any more. On the other hand, if we assume that this data cleaning was done sensibly, there seems to be no reason, a priori, why the estimates should be much worse than they would have been otherwise.

We need some notation. $X_{1}, X_{2}, \ldots, X_{n}$ will denote independent and identically distributed random variables with mean zero, finite variance and a common density $f$, which is symmetric about zero. The standard normal density will be denoted by $\phi$. Let $X_{1: n}<X_{2: n}<\ldots<X_{n: n}$ be the ordered sample and define the trimmed means and their variances by

$$
\bar{X}_{n, k}=\frac{1}{n-2 k} \sum_{i=k+1}^{n-k} X_{i: n}
$$




$$
\sigma_{n, k}^{2}=E \bar{X}_{n, k}^{2} .
$$

For $f=\phi, n=24$ and $k=0,1,2, \ldots, 11$, Hertzsprung estimates the quantities $n \sigma_{n, k}^{2}=\sigma_{n, k}^{2} / \sigma_{n, 0}^{2}$ by the corresponding ratios of the sampling variances of 1000 replications. His results are given in Table 1.

Table 1. HeRTZSPRUNG's estimates of $n \sigma_{n . k}^{2}$ for $f=\phi, n=24, k=0,1, \ldots, 11$.

\begin{tabular}{|c|c||c|c||c|c|}
\hline$k$ & $s_{n, k}^{2} / s_{n, 0}^{2}$ & $k$ & $s_{n, k}^{2} / s_{n, 0}^{2}$ & $k$ & $s_{n, k}^{2} / s_{n, 0}^{2}$ \\
\hline 0 & 1.000 & 4 & 1.095 & 8 & 1.283 \\
1 & 1.013 & 5 & 1.139 & 9 & 1.345 \\
2 & 1.037 & 6 & 1.184 & 10 & 1.407 \\
3 & 1.069 & 7 & 1.232 & 11 & 1.489 \\
\hline
\end{tabular}

Commenting on these numbers, HeRTzSPRUNG writes: "While the cancelling of two arbitrary observations out of 24 diminishes the weight from 24 to 22 the symmetrical rejection of the two largest deviations leaves a weight of nearly 23.7 for the mean of the rest. Hence there is not much reason for hesitation to do so, while the question is still left open as to how false the assigned weights must be in order to obtain an increase of weight by the procedure considered." The word "weight" is used for the reciprocal of the variance and what we have here is a plea for the $5 \%$ trimmed mean!

Finally, HERTZSPRUNG notes that the formula

$$
n \sigma_{n, k}^{2}=1+.53(2 k / n)^{3 / 2}
$$

fits the data in Table 1 quite well. Since the median has asymptotic variance $\pi /(2 n)$ in the normal case, he proposes to replace .53 in (3) by $\pi / 2-1=.57$. The entire paper doesn't take more than one page.

\section{M-estimators}

After attending a talk given by HERTZSPRUNG about his sampling experiment, VAN DE HULST decided to try and treat the problem of finding $\sigma_{n, k}^{2}$ mathematically. In letters to HERTZSPRUNG of April 15 and June 10,1942, he computes values of $\sigma_{24,11}^{2}$ and $\sigma_{24,1}^{2}$. Since these computations also occur in his notes, I shall return to them later. In the weeks that followed, he apparently made quick progress and he wrote down his results in a notebook dated July 1942; there is a supplement dated October 1942. The notes are written in Dutch and are entitled "Over een probleem uit de waarschijnlijkheidsrekening" (On a problem in probability theory). In discussing these notes and other writings, I shall change the notation and terminology to one that is more common in statistics nowadays. Whenever direct quotes occur, the translation is mine.

Since the stated purpose of the notes is to compute the variance of the trimmed 
mean, it is surprising that the author should start with the asymptotic variance of an $M$-estimator:

\section{"Theorem.}

If $n$ observations $X_{i}$ ( $n$ very large) are distributed according to the symmetric probability (density) $f$, and one determines the number $M$ by

$$
\sum_{i=1}^{n} \psi\left(X_{i}-M\right)=0,
$$

where $\psi$ is some odd function, then

$$
E M^{2}=\frac{\int \psi^{2}(x) f(x) d x}{n\left\{\int f(x) d \psi(x)\right\}^{2}} . "
$$

VAN DE HULST calls this result well known and refers to page $470 \mathrm{ff}$ of $\mathrm{F}$. ZERNIKE's (1928) chapter on Probability Theory and Mathematical Statistics in Volume III of the Handbuch der Physik. The amazing implication is that the concept of an $M$-estimator as well as the expression for its asymptotic variance were known to physicists as early as 1928 and it seems worthwhile to take a look at this reference.

The fact that ZERNIKE, a Dutch physicist and a Nobel laureate a quarter of a century later, was asked to write on probability and statistics for the Handbuch, shows that he was considered an authority in this field by his colleagues, and indeed he did give a very interesting and up-to-date account of the area. His treatment of $M$-estimators starts with assuming a large number of observations and a symmetric error density $f$ because (translating his German text) "for a skew error density a sharp distinction between systematic and random errors is not possible" and continues: "The commonly used best (summary) value of $n$ observations is the arithmetic mean, determined by the equation $\Sigma\left(X_{i}-\bar{X}\right)=0$. One considers the generalization of this equation $\Sigma \psi\left(X_{i}-M\right)=0$, where $\psi$ is an odd function of the argument $(x-M)$. This equation can be interpreted as follows: $M$ is the mean of the $X$-values computed with weights $\psi /(X-M)$, i.e. with a symmetric weight function. If the number of $X$-values in every interval would be exactly equal to the (theoretical frequencies) calculated from the error law, then one would find $M=m$ (the expectation of $X$ ). From the statistical deviations of these numbers, one calculates for the deviation of $M$

$$
\sigma^{2}(M)=\frac{\int \psi^{2}(x) f(x) d x}{n\left\{\int f(x) d \psi(x)\right\}^{2}},
$$

and for special choices of the function $\psi$ this formula leads easily to the following results: 


$$
\begin{array}{lll}
\text { Arithmetic mean } & \psi(x)=x & \sigma^{2}=n^{-1} \sigma^{2}(x) \\
\text { Median } & \psi(x)=\operatorname{sgn}(x) & \sigma^{2}=\left(4 n f^{2}(0)\right)^{-1} \\
\text { Best determination } & \psi(x)=f^{\prime}(x) / f(x) & \sigma^{2}=\left(n \int\left(f^{\prime}(x)\right)^{2} / f(x) d x\right)^{-1} . "
\end{array}
$$

ZERNIKE then goes on to provide the variances of these three estimators for four selected error densities. There is no further proof and regularity conditions are not mentioned. He does point out that for the median, the function $\psi$ is not continuous and that the integral in the denominator of (6) should be interpreted as a Stieltjes integral. The interpretation of an $M$-estimator as a weighted mean with random weights is still part of the folklore in this field (cf. HuBER (1981), p. 44). The original of the result (6) is not clear as ZERNIKE doesn't provide any references but doesn't claim the result as his own either. What is clear, is that up-to-date knowledge of statistics did exist in the Netherlands in 1928.

But let us return to the notes of VAN DE HULST. Since there is no proof of the theorem in ZERNIKE's review paper, VAN DE HULST gives one. He prefaces his proof by the remark that the won't be bothered with details, and perhaps this is just as well. It doesn't make much sense to discuss regularity conditions if it isn't even clear what the conclusion of the theorem ought to be. As it stands, it is a statement about the limit of the variance of $n^{1 / 2} M$, as opposed to the variance of the limit distribution of $n^{1 / 2} M$. I doubt that, at the time, many people knew there was a difference between the two, and it certainly wasn't a distinction that was commonly made. We now realize, however, that the former type of result is usually harder to prove, but also less relevant than the latter, because asymptotic theory and small sample approximations concern distributions rather than moments.

Let us then ignore this distinction and look at VAN DE HULST's proof. He starts with the one-term Taylor expansion

$$
0=\sum \psi\left(X_{i}-M\right)=\sum \psi\left(X_{i}\right)-M \sum \psi^{\prime}\left(X_{i}\right)
$$

and rewrites it as

$$
M=\frac{\sum \psi\left(X_{i}\right)}{\sum \psi^{\prime}\left(X_{i}\right)} .
$$

From here on one could argue that $n^{-1 / 2} \Sigma \psi\left(X_{i}\right)$ is asymptotically normal with mean zero and variance $\int \psi^{2} f$ and that $n^{-1} \Sigma \psi^{\prime}\left(X_{i}\right)$ converges in probability to $\int \psi^{\prime} f$ if both integrals are finite. It follows by Slutsky's theorem that (8) implies that $n^{1 / 2} M$ is asymptotically normal with mean zero and variance given by (5). The convergence of the variance of $n^{1 / 2} M$ would take a bit more work.

Instead, VAN DE HULST follows a more devious route that was much travelled in those days. He discretizes the $X^{\prime} s$ by partitioning the real line into a large number of small intervals. If $N_{j}$ of the $X^{\prime} s$ fall in the $j$-th interval and $\psi_{j}$ and $\psi_{j}^{\prime}$ are values which $\psi$ and $\psi^{\prime}$ assume somewhere in this interval, then approximately

$$
M=\frac{\sum N_{j} \psi_{j}}{\sum N_{j} \psi_{j}^{\prime}}
$$


Another Taylor expansion with respect to the $N_{j}$ around $E N_{j}$, followed by the computation of variances and covariances for the multinomial distribution and a passage to the limit, produces. (5).

Of course it is not important how one proceeds from (8) on. The main problem is what happened to the remainder term in (7). To VAN DE HULST this might be a detail not to be worried about, but for VAN DANTZIG it was too much to swallow. In his letter of February 19, 1943, he writes: "It is really a pity that your discussion is so inexact, as this takes away much of the value of the several nice ideas that it contains. For instance, in the "proof" on page 3, I can understand the passage to $0=\Sigma \psi\left(X_{i}-M\right)=\Sigma \psi\left(X_{i}\right)-M \Sigma \psi^{\prime}\left(X_{i}\right)$ only if (1) $\psi(x)$ is differentiable and (2) $M$ is small, whereas $\psi^{\prime \prime}(x)$ remains bounded (even then I don't see how one can know in advance that the term involving $M^{2}$ can be neglected, since it is the calculation of $M^{2}$ we are concerned with)."

VAN DANTZIG is clearly right in pointing out that this kind of proof will work only for smooth functions $\psi$ and I'm sure that VAN DE HULST readily agreed. It is interesting, however, that VAN DANTZIG had difficulty seeing how one shows that $M$ is small, i.e. that $M$ is a consistent estimator. If, in addition to its smoothness, one simply assumes $\psi$ to be nondecreasing and strictly increasing on a set of positive probability under $f$, then $n^{-1} \Sigma \psi\left(X_{i}-m\right)$ is a continuous and nonincreasing function of $m$ and $\int \psi(x-m) f(x) d x$ is strictly decreasing in $m$ in a neighborhood of zero. The consistency of $M$ now follows from the law of large numbers in the same way as in CrAMÉR's (1945) proof of the consistency of the maximum likelihood estimator. Of course CRAMER's book had not yet appeared, and apparently this argument was not yet generally known.

Having completed his proof, VAN DE HULST mentions the special cases of the mean and the median discussed by ZERNIKE, but again VAN DANTZIG is not impressed: "But under no circumstances can I understand how this proof can be applied to $\psi(x)=\operatorname{sgn}(x)$. What is the meaning of $M=\Sigma \psi\left(X_{i}\right) / \Sigma \psi^{\prime}\left(X_{i}\right)$ here? Therefore, these considerations don't prove anything for the case of the median."

Let us put these mathematical objections aside for a moment and take stock of what has been archieved so far. An expression has been derived for the asymptotic variance of an $M$-estimator of location. The proof is rather shaky, but we know today that the expression does indeed hold in great generality, including the case of the sample median. The reason is, that it is the smoothness of $\lambda(m)=E \psi(X-m)$ which is important rather than the smoothness of $\psi$. However, the original purpose was to find the asymptotic variance of the trimmed mean. Of course the two extreme cases of the trimmed mean, the sample median and the untrimmed mean, are also $M$-estimators but their asymptotic variances were already known. VAN DE HULST writes: "One wonders whether the intermediate cases (of the trimmed mean) ... can also be written in the form $\Sigma \psi\left(X_{i}-M\right)=0$ by choosing an appropriate function $\psi$. However, this is not the case and the result obtained above can not he used directly. Nevertheless it turns out that with a minor modification in the above proof, this case can also be treated." 


\section{Trimmed means}

As before, let $X_{1}, \ldots, X_{n}$ be independent and identically distributed with mean zero, finite variance and a common density $f$, which is symmetric about zero. The distribution function corresponding to $f$ is denoted by $F$. Consider the trimmed mean $\bar{X}_{n, k}$ and its variance $\sigma_{n, k}^{2}$ defined by (1) and (2). Denote the trimming fraction on each side by $\alpha$ and let $a$ be the upper $\alpha$-point of $F$, thus

$$
\alpha=\frac{k}{n}, \quad F(a)=1-\alpha .
$$

Define the function $\psi_{0}$ by

$$
\psi_{0}(x)=\left\{\begin{aligned}
-a & \text { if } \quad x<-a, \\
x & \text { if }-a \leqslant x \leqslant a, \\
a & \text { if } x>a .
\end{aligned}\right.
$$

VAN DE HULST shows that if $n \rightarrow \infty$ and $\alpha$ remains bounded away from $\frac{1}{2}$, then

$$
n \sigma_{n, k}^{2} \sim(1-2 \alpha)^{-2} \int \psi_{0}^{2}(x) f(x) d x,
$$

where $\sim$ denotes asymptotic equality. I shall try to give a simplified version of VAN DE HULST's argument which, I hope, still retains the original flavor. An entirely different proof of (12) was given earlier in DaNiELL (1920), but this paper seems to have gone completely unnoticed (cf. STIGLER (1973)).

Define the interval $I=\left(X_{k: n}, X_{n-k: n}\right.$ and let $F_{n}$ be the empirical distribution function. We can write

$$
\bar{X}_{n, k}=(1-2 \alpha)^{-1} \int_{I} x d F_{n}(x)
$$

Neglecting lower order terms, we have

$$
\begin{gathered}
\alpha-F_{n}(-a)=F_{n}\left(X_{k: n}\right)-F_{n}(-a) \sim\left(X_{k: n}+a\right) f(a), \\
1-\alpha-F_{n}(a)=F_{n}\left(X_{n-k: n}\right)-F_{n}(a) \sim\left(X_{n-k: n}-a\right) f(a) .
\end{gathered}
$$

This is intuitively clear, but not entirely trivial to prove rigorously. It is called the BAHADUR representation after BAHADUR (1966), who proved that the remainder term is $O\left(n^{-3 / 4} \log n\right)$ almost surely. Similarly,

$$
\int_{I} x d F_{n}(x)-\int_{-a}^{a} x d F_{n}(x) \sim\left(X_{k: n}+X_{n-k: n}\right) a f(a) .
$$

Combining (13), (14), (15) and (11), we find

$$
\bar{X}_{n, k} \sim(1-2 \alpha)^{-1} \int \psi_{0}(x) d F_{n}(x)=(1-2 \alpha)^{-1} n^{-1} \sum \psi_{0}\left(X_{i}\right)
$$

and with a little bit of luck this implies (12).

Since $\int f d \psi_{0}=(1-2 \alpha)$, a comparison of (6) and (12) shows that the asymptotic 
variances of the $\alpha$-trimmed mean and the $M$-estimator with $\psi=\psi_{0}$ (which is known as HUBER's estimator) coincide. Of course VAN DE HULST is pleased to note this and it confirms his intuition that $M$-estimators would have something to do with the problem. On the other hand, however, he finds the agreement of the two variances rather fortuitous.

Photograph 1. Part of a page in VAN DE HULST's notebook where he concludes that the trimmed mean has the same asymptotic variance as HUBER's estimator. Note the drawing of the influence curve of these two estimators, which is almost a symbol of robust statistics. The scribbled line at the bottom is VAN DANTZIG's.

Opmertaing. Achterafbligter, dat dese nitkomst. toch vom clescefte vorm is, als zon verkrequs waden met een h-fmuctic volgens helbetende recept (ble 3) van nevenstacade vorm.

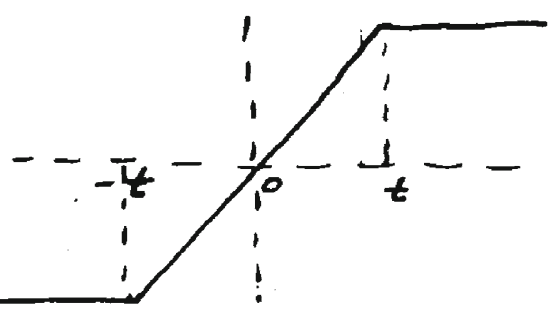

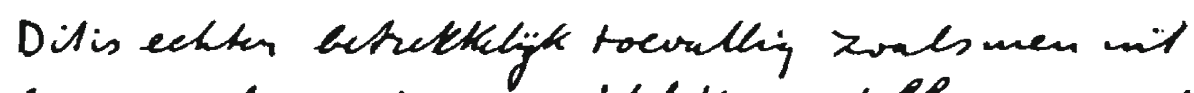

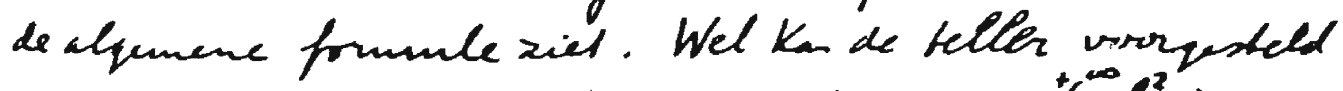
worden dovr en intersad $v d$. vereiste vorm: $\int_{-\infty}^{+\infty} h^{2} d x$ (de factor $\left(\frac{h_{0}}{k_{0}}\right)^{2}$ betetend continue arushiting),

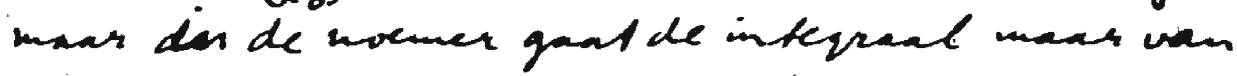
$-t+o t+t$. Toevallij ecther is $k(x)=g y(x)$, dins $d k=0$

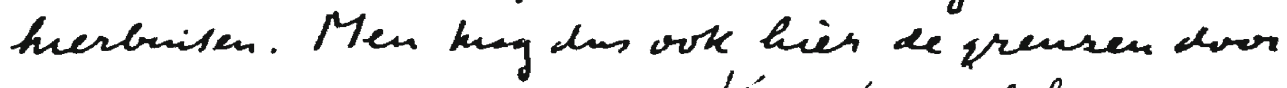
$-\infty$ en $+\infty$ verurangen. K'an stedis al finctie $w$

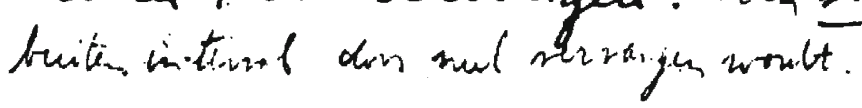

A closer inspection of his argument, however, shows that the agreement goes much further than he may have realized. As I remarked below (8), we have $n^{-1} \Sigma \psi^{\prime}\left(X_{i}\right) \sim \int \psi^{\prime} f$ and hence

$$
M \sim \frac{\Sigma \psi\left(X_{i}\right)}{n \int \psi^{\prime}(x) f(x) d x} .
$$


Choosing $\psi=\psi_{0}$, we find for HUBER's estimator $H$,

$$
H \sim(1-2 \alpha)^{-1} n^{-1} \sum \psi_{0}\left(X_{i}\right),
$$

and together, (16) and (18) imply that $n^{1 / 2}\left(\bar{X}_{n, k}-H\right)$ tends to zero in probability. Thus HUBER's estimator and the $\alpha$-trimmed mean are asymptotically equivalent estimators and the equality of the asymptotic variances is merely a consequence of this.

There is an interesting discussion connected with this result. When robust estimators began to be investigated, it was felt by some, that throwing away observations, as one does when computing a trimmed mean, is perhaps overdoing things a little. Wouldn't it be better to move these observations towards the center of the sample and replace them by $X_{k+1: n}$ and $X_{n-k: n}$ rather than deleting them outright? This led to the introduction of the so-called Winsorized mean

$$
W_{n, k}=\frac{1}{n}\left[\sum_{i=k+1}^{n-k} X_{i: n}+k\left(X_{k+1: n}+X_{n-k: n}\right)\right] \text {. }
$$

Later, after HUBER's estimator had been introduced and found to perform well, representation (18) and the shape of $\psi_{0}$ as given by (11) seemed to suggest that the same thing is going on here: the $X_{i}$ are replaced by $\psi_{0}\left(X_{i}\right)$, which moves the outlying observations towards the center. On the strength of this, it was assumed that the Winsorized mean would mimic HUBER's estimator and share its good performance to a greater extent than the trimmed mean. This argument is not really very convincing because, in view of the symmetry of $f$, the effect of moving observations towards the center depends very much on the exact number of observations moved and the exact positions they are moved to. Nevertheless, this idea was rather generally accepted until BiCKEL (1965) proved that the asymptotic distribution of the trimmed mean is the same as that of HUBER's estimator. In HuBER's words (cf. HUBER (1981), p. 59): "This exemplifies how unreliable our intuition can be; we know now ... that the trimmed mean does not throw away all of the information sitting in the discarded observations, but that it does exactly what the Winsorized mean was supposed to do." Of course one can agree with this sentiment in general, but after seeing VAN DE HULST's notes, one is inclined to add that this depends very much on whose intuition one is talking about!

For $f=\phi,(12)$ reduces to

$$
n \sigma_{n, k}^{2} \sim(1-2 \alpha)^{-2}\left\{(1-2 \alpha)-2 a \phi(a)+2 \alpha a^{2}\right\}
$$

and for $n=24$, these values are given in Table 2 . 
Table 2. Asymptotic values of $n \sigma_{n, k}^{2}$ for $f=\phi, n=24, k=0,1, \ldots, 11$.

\begin{tabular}{|c|c||c||c||c|c|}
\hline$k$ & $n \sigma_{n, k}^{2}$ & $k$ & $n \sigma_{n, k}^{2}$ & $k$ & $n \sigma_{n, k}^{2}$ \\
\hline 0 & 1.000 & 4 & 1.114 & 8 & 1.294 \\
1 & 1.021 & 5 & 1.153 & 9 & 1.352 \\
2 & 1.048 & 6 & 1.195 & 10 & 1.417 \\
3 & 1.079 & 7 & 1.242 & 11 & 1.483 \\
\hline
\end{tabular}

These figures seem to agree reasonably well with HERTZSPRUNG's empirical data in Table 1. Commenting on VAN DE HULST's further efforts to improve the asymptotic approximations, VAN DANTZIG writes: "On the whole, I don't think it makes sense to try to explain the small deviations between your calculations and HERTZSPRUNG's results; in my opinion, the agreement is too good to be true." $\mathrm{He}$ argues that, since HERTZSPRUNG's data have been "doctored", one certainly can't attach "any value to the third, and perhaps even to the second decimal of the empirical data".

In view of the agreement which is "too good to be true", it is perhaps not surprising that VAN DANTZIG also feels that (20) is "almost certainly incorrect". The reason for his doubts is interesting. Apart from the argument for (12) and (20) that we have just sketched, VAN DE HULST also provides a second proof of (20), which is precisely the proof that one would give today: Given $X_{k: n}$ and $X_{n-k+1: n}$, the trimmed mean is distributed as an ordinary sample mean and the conditional second moment is easily calculated. Taking the expectation with respect to the bivariate normal limit distribution $\left(X_{k: n}+a\right)$ and $\left(X_{n-k+1: n}-a\right)$, one obtains (20). VAN DANTZIG argues, mistakenly, that second order terms of this bivariate distribution should also play a role and give rise to additional terms in the final result. The fact that the two proofs produce the same result, merely leads him to conclude that the first proof is probably incorrect too.

Van Dantzig's letter from which I have quoted repeatedly, was typical for the person I believe he was. His criticism was very much to the point and mathematically correct, except for his doubts about the validity of (20). It was offered in a matter-of-fact way and it probably didn't occur to him at first, that it might have a rather devastating effect on the receiver of the letter. He was genuinely trying to help and felt hat the best way to do this, was to explain his views as clearly as possible. 
Photograph 2. End of VAN DANTzig's letter to VAN DE Hulst.

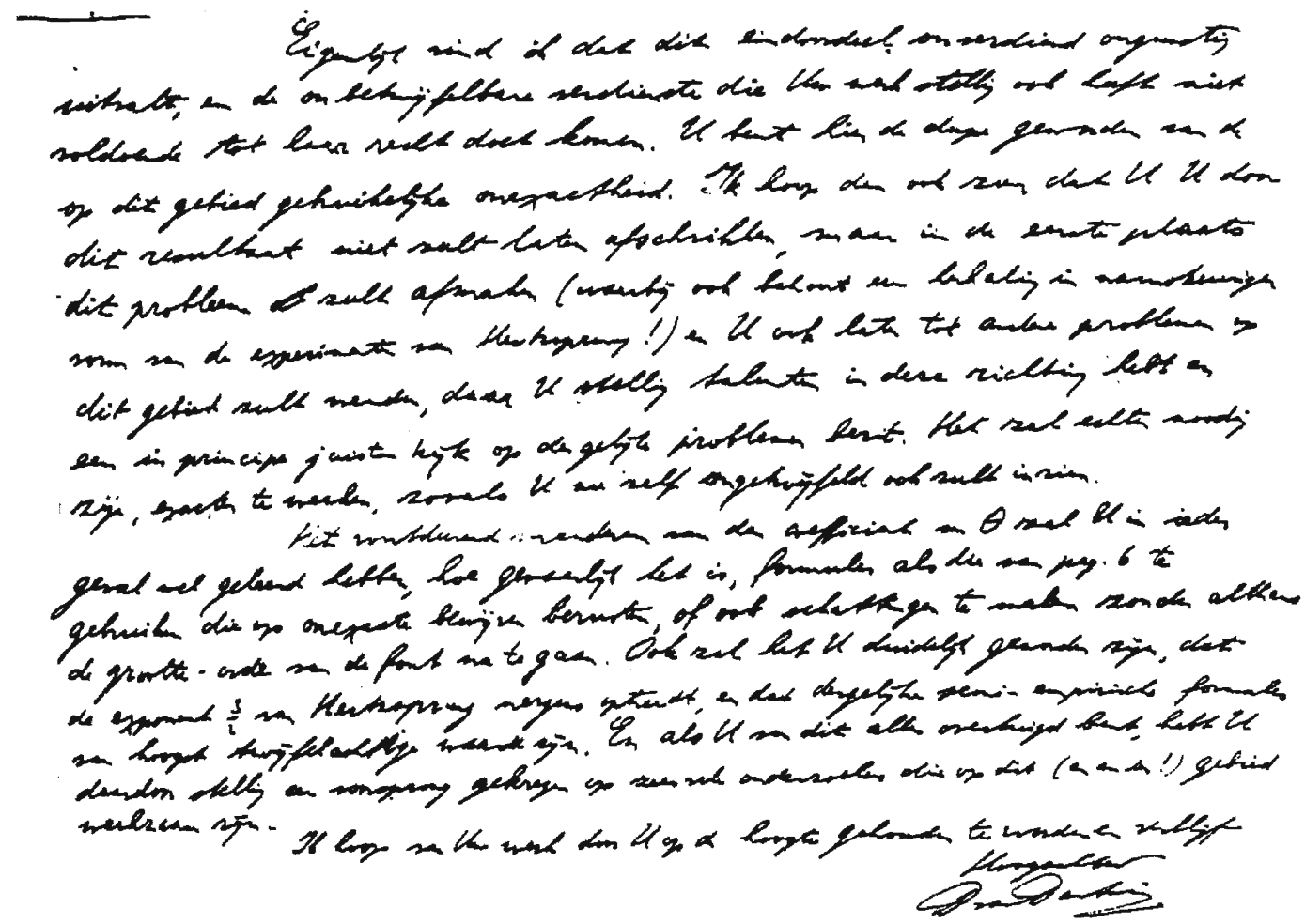

I imagine that it was when rereading the letter, that he felt that perhaps he had been a bit too severe. He was basically a kind person and he could certainly recognize talent when he saw it. He added: "On second thought I find that my final opinion has turned out undeservedly unfavorable and that it doesn't do justice to the indubitable merit of your work. ... I do hope that you won't be discouraged by this result and that you'll first finish this problem ... and then turn to other problems in this area. You definitely have talents in this direction and in principle you have the right way of looking at such problems." Of course this praise was interspersed with admonitions to work more rigorously!

\section{Other problems}

As was mentioned in section 3, VAN DE HULST started out by studying the median $X_{n}$, which he defined in the usual way as

$$
\begin{array}{lll}
X_{\frac{1}{2}(n+1): n} & =\bar{X}_{n, \frac{1}{2}(n-1)} \text { if } n \text { is odd, } \\
\tilde{X}_{n}=\frac{1}{2}\left(X_{\frac{1}{2} n: n}+X_{\frac{1}{2} n+1: n}\right) & =\bar{X}_{n, \frac{1}{2} n-1} \text { if } n \text { is even. }
\end{array}
$$


It was known that for $f=\phi$, the asymptotic variance of $n^{1 / 2} \bar{X}_{n}$ equals $\frac{1}{2} \pi$. VAN DE HULST tried to calculate a correction term of order $n^{-1}$. After several unsuccessful attempts, both for odd an even $n$, the finally found a series expansion for odd $n$ and $f=\phi$,

$$
n \sigma^{2}\left(\tilde{X}_{n}\right)=\frac{\pi}{2}\left[1-\frac{4-\pi}{2 n}-\frac{3 \pi-4}{n^{2}}+\frac{13 \pi^{2}}{24 n^{2}}+\ldots\right] .
$$

Unfortunately this result can't be used for $n=24$, and the expansion for even $n$ is harder to obtain.

He also tried to improve the approximation for $\sigma_{n, 1}^{2}$. Writing

$$
M_{n}=\frac{1}{2}\left(X_{1: n}+X_{n: n}\right)
$$

for the midrange, VAN DE HULST derives an exact relation between the variances of the trimmed mean $\bar{X}_{n, 1}$, the mean $\bar{X}_{n, 0}$ and the midrange $M_{n}$, for the case $f=\phi$,

$$
\frac{\sigma_{n, 1}^{2}}{\sigma_{n, 0}^{2}}-1=\frac{4}{(n-2)^{2}}\left[\frac{E M_{n}^{2}}{\sigma_{n, 0}^{2}}-1\right] \text {. }
$$

By numerical methods he found $\sigma^{2}\left(M_{n}\right) / \sigma_{n, 0}^{2}=3.22$ for $n=24$ and $f=\phi$, and this yields $n \sigma_{n, 1}^{2}=1.018$. He concludes that this is probably a better estimate than the value 1.013 found by HERTZSPRUNG, especially since the estimate of $\sigma^{2}\left(M_{n}\right) / \sigma_{n, 0}^{2}$ from HERTZSPRUNG's sampling data equals 3.212.

So far for VAN DE HULST's work on HERTZSPRUNG's problem. However, the final insight was still to come. On December 21, 1943, more than a year later, he writes to HERTZSPRUNG: "And now a few words about the calculation of the means of the proper motions of the Pleiades. You found empirically that the variance of the trimmed mean of 24 observations with a Gaussian distribution depends on $\alpha=k / n$ according to curve (a) (an increasing function of $\alpha$ is shown). I later found practically the same result by computation. In connection with the things you showed me on December 1 last, I discovered yet another possibility. It is possible to apply my formula (Formula (12) not to a Gaussian distribution, but to the true distribution of the measurement errors, including the so-called outliers. In a fictitious example I obtained curve (b) (a function of $\alpha$ which first decreases rapidly, and then increases more slowly, is shown). On this curve one can read off precisely how much the variance of the mean decreases, if one rejects one or more observations symmetrically. The location of the minimum in this example indicates, that it is best to reject about $25 \%$ of the observations, that is 3 on both sides out of 24 !" He then proposes to estimate the distribution of the errors in HeRTZSPRUNG's sampling experiment and find the optimal trimming percentage.

Presumably things never got to that point, but the computation to which VAN DE HULST refers is attached to the notebook. It concerns an error distribution with a range from -4.24 to +4.24 and his conclusion is that about $10 \%$ should be trimmed on both sides. Here he is definitely on the road that TUKEY would take in 
$1949 !$

\section{Can we do better now?}

It may be of interest to consider briefly how we would deal with this problem today and ask whether we could do essentially better. I believe the answer is: not very much. Of course we can now prove these results rigorously and in great generality. Also, we have sufficient computing power at our disposal to compute the required quantities exactly, if need be. It is a good thing that VAN DE HULST didn't have that possibility, because he wouldn't have discovered anything if he had.

The first thing we can do is to compute the asymptotic variance of HERTZSPRUNG's estimator of $n \sigma_{n, k}^{2}$,

$$
\frac{s_{n, k}^{2}}{s_{n, 0}^{2}}=\frac{\sum_{j=1}^{N}\left\{\bar{X}_{n, k}^{(j)}\right\}^{2}}{\sum_{j=1}^{N}\left\{\bar{X}_{n, 0}^{(j)}\right\}^{2}},
$$

where $\bar{X}_{n, 0}^{(j)}$ and $\bar{X}_{n, k}^{(j)}, j=1, \ldots, N$, denote the means and trimmed means of the $N=1000$ samples of size $n=24$. For $f=\phi$ we have, as $n, N \rightarrow \infty$,

$$
\sigma^{2}\left(\frac{s_{n, k}^{2}}{s_{n, 0}^{2}}\right)=\frac{4 n \sigma_{n, k}^{2}}{N}\left(n \sigma_{n, k}^{2}-1\right)+\Theta\left(\frac{1}{N n}+\frac{1}{N^{2}}\right),
$$

where we may replace $n \sigma_{n, k}^{2}$ by the right-hand side of (20). Of course, one could also use $n s_{n, k}^{2}$ as an estimate for $n \sigma_{n, k}^{2}$, and then we find, for $f=\phi$,

$$
\sigma^{2}\left(n s_{n, k}^{2}\right)=\frac{2 n^{2} \sigma_{n, k}^{4}}{N}+\mathcal{O}\left(\frac{1}{N n}+\frac{1}{N^{2}}\right) \text {. }
$$

This confirms what was already intuitively obvious, that HeRTZSPRUNG's estimator is better than $n s_{n, k}^{2}$, especially for small values of $\alpha=k / n$, when $n \sigma_{n, k}^{2}$ is close to 1 .

Another thing we can do is to calculate a second order approximation for $n \sigma_{n, k}^{2}$. Such approximations, including a term of order $n^{-1}$, can be found in HeLmers (1982) for general linear combinations of order statistics. However, use of these general formulas involves very lengthy computations and in this simple case, it is easier to start from scratch. In the notation of Section 4 we find, for $f=\phi$ and $\alpha$ bounded away from $\frac{1}{2}$,

$$
\begin{aligned}
n \sigma_{n, k}^{2}= & \frac{1}{(1-2 \alpha)^{2}}\left[(1-2 \alpha)-2 a \phi(a)+2 \alpha a^{2}+n^{-1}\left\{-2 \alpha(1-\alpha) \frac{a}{\phi(a)}\right.\right. \\
& \left.\left.+2 \alpha^{2}(1-\alpha)\left(\frac{a}{\phi(a)}\right)^{2}+\alpha^{2}(1-2 \alpha) \frac{1}{(\phi(a))^{2}}\right\}\right]+O\left(n^{-2}\right) .
\end{aligned}
$$


For easy comparison Table 3 provides HeRTZSPRUNG's estimates of $n \sigma_{n, k}^{2}$ taken from Table 1 , the asymptotic standard deviation of these estimates computed from (26) and (20), the first order approximation of $n \sigma_{n, k}^{2}$ taken from Table 2 and finally the second order approximation of $n \sigma_{n, k}^{2}$ computed from (28).

Table 3. HERTZSPRUNG's estimate, its standard deviation and approximate values of $n \sigma_{n, k}^{1}$ for $f=\phi, n=24, k=0,1, \ldots, 11$.

\begin{tabular}{|r|c|c|c|c|}
\hline$k$ & $s_{n, k}^{2} / s_{n, 0}^{2}$ & $\sigma\left(s_{n, k}^{2} / s_{n, 0}^{2}\right)$ & $\begin{array}{c}n \sigma_{n, k}^{2} \\
\text { first order }\end{array}$ & $\begin{array}{c}n \sigma_{n, k}^{2} \\
\text { second order }\end{array}$ \\
\hline 0 & 1.000 & 0 & 1.000 & 1.000 \\
1 & 1.013 & .009 & 1.021 & 1.017 \\
2 & 1.037 & .014 & 1.048 & 1.043 \\
3 & 1.069 & .019 & 1.079 & 1.073 \\
4 & 1.095 & .023 & 1.114 & 1.106 \\
5 & 1.139 & .027 & 1.153 & 1.143 \\
6 & 1.184 & .031 & 1.195 & 1.185 \\
7 & 1.232 & .035 & 1.242 & 1.230 \\
8 & 1.283 & .039 & 1.294 & 1.280 \\
9 & 1.345 & .044 & 1.352 & 1.335 \\
10 & 1.407 & .049 & 1.417 & 1.397 \\
11 & 1.489 & .054 & 1.483 & 1.459 \\
\hline
\end{tabular}

Inspection of this table shows that the agreement between HERTZSPRUNG's estimates and the asymptotic values is closer than one would expect; the difference never exceeds the standard deviation of the estimate and is considerably smaller in most cases. Perhaps HerTzSPRUNG's data cleaning worked rather well!

The second order approximation looks somewhat better than the first order approximation, except at the bottom of the table. This is as it should be. The first order result (20) is valid for all values of $\alpha=k / n$, but the second order result (28) holds only if $\alpha$ is bounded away from $1 / 2$ as $n$ tends to infinity. For values of $\alpha$ close to $1 / 2$, it can therefore be expected to give a bad approximation. For $k=11$, it would be better to use the second order approximation for the median for even sample size, which VAN DE HULST was unable to find. For even $n$ and $f=\phi$, it is

$$
n \sigma^{2}\left(\tilde{X}_{n}\right)=\frac{\pi}{2}\left(1-\frac{6-\pi}{2 n}\right)+\theta\left(n^{-2}\right)
$$

and for $n=24$, this yields 1.477 . 


\section{References}

BaHadur, R.R. (1966), A note on quantiles in large samples, Ann. Math. Statist. 37, 577-580.

BICKEL, P.J. (1965), On some robust estimates of location, Ann. Math. Statist. 36, 847-858.

CRAMER, H. (1945), Mathematical Methods of Statistics, Princeton University Press, Princeton.

DANiel.l, P.J. (1920), Observations weighted according to order, American Journal of Mathematics 42, 222-236.

HeLmers, R. (1982), Edgeworth Expansions for Linear Combinations of Order Statistics, Mathematical Centre Tracts 105, Mathematisch Centrum, Amsterdam.

Hemelrux, J. (1959), In memoriam Prof.Dr. D. van Dantzig, Statistica Neerlandica 13, 416-432.

HertzsPrung, E. (1942), On the symmetrical rejection of extreme observations, Bulletin of the Astronomical Institutes of the Netherlands IX (349), 285-286.

Huber, P.J. (1981), Robust Statistics, Wiley, New York.

Stigler, S.M. (1973), Simon Newcomb, Percy Daniell, and the history of robust estimation 1885-1920, J. Amer. Statist. Assoc. 68, 872-879.

ZERNIKE, F. (1928), Wahrscheinlichkeitsrechnung und mathematische Statistik. In: Handbuch der Physik, Bd III, Mathematische Hilfsmittel in der Physik, Kap. 12, Springer, Berlin.

Dept. of Mathematics and Computer Science

University of Leiden

P.O. Box 9512

2300 RA Leiden

The Netherlands 